\title{
THE VELOCITY DISPERSIONS OF THE DRACO AND URSA MINOR DWARF SPHEROIDAL GALAXIES
}

\section{PRYOR}

Rutgers, the State Univ. of New Jersey

Dept. of Phys. \& Astron., P.O. Box 849, Piscataway, NJ 08855

E. W. OLSZEWSKI

Steward Observatory

Univ. of Arizona, Tucson, $A Z 85721$

\section{AND}

\section{T. E. ARMANDROFF}

\section{NOAO}

Kitt Peak National Obs., P.O. Box 26732, Tucson, AZ 85726

We have used the Hydra fiber positioner and the bench spectrograph on the KPNO $4 \mathrm{~m}$ telescope to measure radial velocities for giants in the Draco (Dra) and Ursa Minor (UMi) dwarf spheroidal galaxies. The measurement uncertainties are $1-10 \mathrm{~km} \mathrm{~s}^{-1}$ per observation, with the median uncertainty being $3.6 \mathrm{~km} \mathrm{~s}^{-1}$. To date, we have reduced the data from two of our three runs. In UMi these have yielded 150 velocities for 85 member giants; 52 stars have two or more measurements. In Dra we have 108 velocities for 84 member giants and 20 stars have two or more measurements. There is good agreement between the repeat observations and with the Olszewski, Aaronson, \& Hill (1995) MMT echelle velocities.

The star N98 in UMi has a velocity of $-297 \pm 4 \mathrm{~km} \mathrm{~s}^{-1}$, while the mean velocity of the rest of the sample is $-245.7 \pm 1.1 \mathrm{~km} \mathrm{~s}^{-1}$. Similarly, star Dra278 has a velocity of $-332.5 \pm 1.4 \mathrm{~km} \mathrm{~s}^{-1}$, with the rest of the Dra sample yielding an average of $-291.9 \pm 1.1 \mathrm{~km} \mathrm{~s}^{-1}$. Both stars are projected near the centers of their galaxies and are near the giant branch in colormagnitude diagrams. If further study confirms membership and constant velocities (we have measurements at only a single epoch), these stars will imply very large total M/L's for UMi and Dra. Excluding these two stars, our UMi and Dra samples yield velocity dispersions of $8.9 \pm 0.8 \mathrm{~km} \mathrm{~s}^{-1}$ and $9.2 \pm 0.8 \mathrm{~km} \mathrm{~s}^{-1}$, respectively. If mass follows light, then the two galaxies 

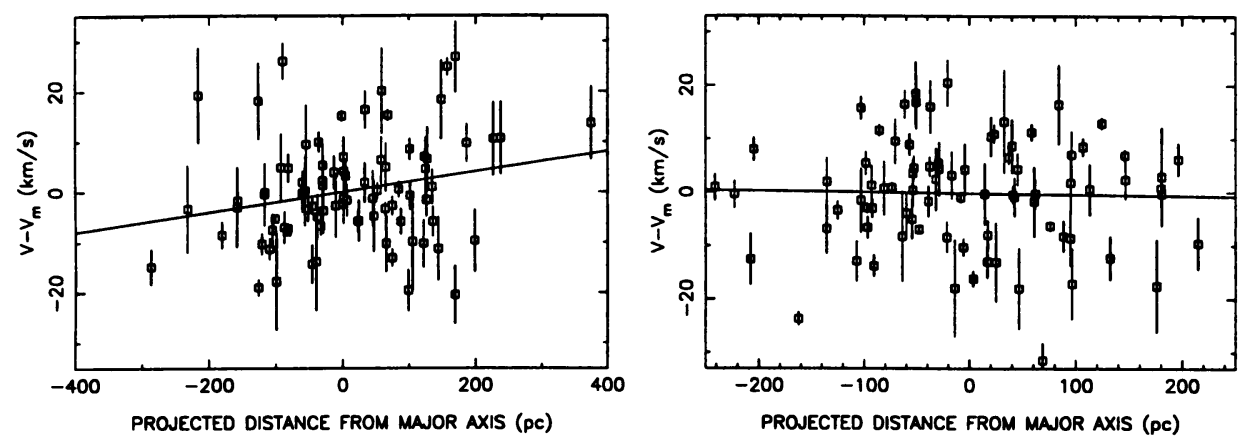

Figure 1. The left panel shows the deviation from the systemi: velocity vs. distance from the major axis for 84 stars in our UMi sample. The line is the slope determined by least squares, which has the parameters given in the text. Note that, while there is a significant velocity gradient, the scatter of the velocities about that gradient is large. The right panel is the same plot for the 82 stars in our Draco sample. The least-squares velocity gradient is effectively zero.

have V-band mass-to-light ratios (M/L's) of $100 \pm 20$ and $110 \pm 20$ in solar units. The quoted uncertainties are those due only to the uncertainties in the dispersion.

We confirm the velocity gradient approximately along the minor axis of UMi seen by Hargreaves et al. (1994), with a sample that is twice as large as theirs. The left panel of Figure 1 plots our velocities (excluding N98) vs. distance from the major axis. Least squares yields a velocity gradient of $2.0 \pm 1.0 \mathrm{~km} \mathrm{~s}^{-1}(100 \mathrm{pc})^{-1}$. The right panel is the same plot for Dra (Dra278 is excluded), for which we find no significant velocity gradient along any axis. The two simplest explanations for the velocity gradient in UMi, rotation and tidal disruption, would both be more likely to produce a gradient along the major axis than the minor axis. Tidal forces produce ordered rather than random velocities (Oh et al. 1994; Piatek \& Pryor $1994)$. Since Fig. 1 shows that the dispersion about the gradient is large $\left(8.5 \mathrm{~km} \mathrm{~s}^{-1}\right)$, even if tides are operating they are not the cause of the large measured $M / L$. If rotation is the cause of the gradient, then our $M / L$, calculated without subtracting any rotation, should be reasonably accurate (if mass follows light).

EO and CP acknowledge support from the NSF through grants AST 92-23967 and AST 90-20685, respectively.

\section{References}

Hargreaves, J.C., Gilmore, G., Irwin, M.J., and Carter, D. 1994 Monthly Notices Roy. Astron. Soc., in press.

Oh, K.S., Lin, D.N.C., and Aarseth, S.J. 1994 Astrophys. J., in press.

Olszewski, E.W., Aaronson, M., and Hill, J.M. 1995 in preparation.

Piatek, S. and Pryor, C. 1994 Astron. J., in press. 